\title{
Validity and reliability of the Turkish version of the insomnia catastrophizing scale
}

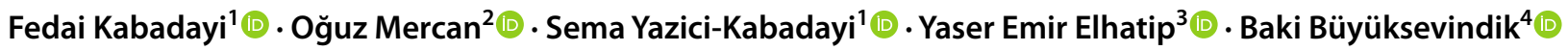

Received: 6 March 2021 / Accepted: 15 June 2021 / Published online: 24 June 2021

(c) Japanese Society of Sleep Research 2021

\begin{abstract}
Insomnia catastrophizing has attracted attention in recent years. Individuals may have started to recognize the importance of evaluating the consequences of insomnia, which has worsened during the coronavirus disease 2019 (COVID-19) pandemic. The present study aimed to test the validity and reliability of the Insomnia Catastrophizing Scale (ICS) in the Turkish young adult population. The study group consisted of a total of 387 participants: 57 in the pilot study and 330 in the main study. The majority of the participants in the study were females. According to the results of the confirmatory factor analysis, the single-factor structures of the ICS nighttime and daytime forms were confirmed to be the same as in the original structure, and the fit indices were above the acceptable limits. The ICS forms had very high reliability. The findings of the present study showed that the Turkish daytime and nighttime forms of the ICS are valid and reliable instruments. The results provide a means of measuring insomnia catastrophizing for use in therapeutic and counseling settings for Turkish young adult population. We also propose that mental health practitioners should design interventions to reduce insomnia catastrophizing in Turkish culture.
\end{abstract}

Keywords Insomnia $\cdot$ Catastrophizing $\cdot$ Validation $\cdot$ Reliability $\cdot$ Factor analysis $\cdot$ Turkish

Fedai Kabadayi

fdkbdy@gmail.com

Oğuz Mercan

oguzmercan26@gmail.com

Sema Yazici-Kabadayi

sema.yazici@erdogan.edu.tr

Yaser Emir Elhatip

yaseremir@gmail.com

Baki Büyüksevindik

baki.sevindik@gmail.com

1 Department of Counseling and Guidance, Recep Tayyip Erdogan University, Faculty of Education, Rize, Turkey

2 Ministry of National Education, Alpu Ortaokulu, Eskişehir, Turkey

3 Department of Counseling and Guidance, Aksaray University, Faculty of Education, Aksaray, Turkey

4 Department of Counseling and Guidance, Bayburt University, Faculty of Education, Bayburt, Turkey

\section{Introduction}

Insomnia is a major psychiatric disorder, and insomnia and insomnia-related disorders have been defined in the DSM-5 [1]. Insomnia has several characteristics, such as difficulty falling asleep and poor-quality sleep [2]. Wide ranges in prevalence rates of insomnia were reported before the COVID-19 pandemic [3]. Sleep disorders and other sleeprelated problems have become increasingly common during the COVID-19 pandemic. Recent findings emphasized the high prevalences of insomnia and insomnia-related problems among the general population [4] and healthcare workers [5]. In the latest study, the prevalence of poor sleep in the Turkish adult population was $55.1 \%$, which is remarkably high [6].

There are some conditions that result in the exacerbation of insomnia in the clinical and non-clinical populations. Insomnia catastrophizing is related to the cognitive aspect of insomnia and is a developing concept that has recently gained attention. Sleep disorders, such as insomnia, are associated with catastrophic consequences [7]. In catastrophizing, which includes making unreasonable and negative predictions [8], the negative consequences of a situation are 
exaggerated. Catastrophizing thoughts that are associated with various psychological and physiological problems may be related to the individual himself, his environment, or the world [9]. Catastrophizing thoughts also include destructive cognitive evaluations of the answer to the question "what if it happens" in relation to a catastrophic situation that causes anxiety [10]. Similarly, insomnia catastrophizing is the cognitive exaggeration of the consequences of sleep disturbance and the resulting impairment of daily functioning [7]. Insomnia catastrophizing is related to negative thoughts about insomnia rather than daily anxieties or poor sleep quality [11]. The insomnia catastrophizing includes sleeprelated worries, pre-sleep cognitive arousal, and general anxiety. Insomnia is also associated with early awakening and a decrease in total sleep time. In insomnia catastrophizing, which is considered in two categories, nighttime and daytime, individuals have catastrophizing thoughts about nighttime insomnia that negatively affect daytime functioning [12].

Some measurements are needed to assess insomnia and related problems. There are several instruments currently available to measure insomnia [13]. The Bergen Insomnia Scale (BIS) [14], Insomnia Severity Index (ISI) [15], Athens Insomnia Scale (AIS) [16], and Catastrophic Thoughts about Insomnia Scale (CTIS) [11] are some of these instruments. Another instrument is the ICS. The ICS is a 17-item self-report questionnaire that assesses both nighttime and daytime insomnia catastrophizing [17]. ICS differs from other insomnia measurement instruments in several ways. First, ICS appears to be able to distinguish individuals with insomnia from those with poor sleep and those who have adequate sleep without a specific disorder [7]. Second, there are psychological effects of insomnia in both the daytime and the nighttime. This situation is expressed based on the cognitive model [18]. Third, similar measurement instruments for insomnia catastrophizing (e.g., CTIS; [11]) focus on past and present rumination, making them theoretically inappropriate for the measurement of catastrophizing, which focuses on the future. The ICS focuses on catastrophizing thoughts about the future rather than about current and past events [12].

The psychometric properties of ICS were examined in a large community-dwelling sample [12], and Italian [7] and Portuguese versions [19] were adapted. The presence of a one-factor structure was confirmed for both the Insomnia Catastrophizing Scale-Nighttime (ICS-N) and the Insomnia Catastrophizing Scale-Daytime (ICS-D). Additionally, the relationship of the ICS with specific variables was also discussed in these studies. For example, in the study on the Italian version of the scale, it was stated that ICS-N and ICS-D scores increased with age, and sex played a mediating role between catastrophizing thoughts and insomnia severity [7]. Instruments measuring insomnia-related cognition are limited in Turkey. Some of the commonly used scales are the Insomnia Severity Index [20], Bergen Insomnia Scale [21] and Pittsburgh Sleep Quality Index [22]. Only one instrument measures catastrophizing, rumination, or cognitive factors in insomnia [23].

The findings of previous studies showed the importance of the control of dysfunctional cognition in cognitively based models of insomnia [24, 25]. In addition, studies have shown that there are several points of association between insomnia and anxiety. It has been reported that general anxiety and insomnia affect daily functioning [26, 27]. Considering the possible relationship of catastrophizing thoughts about insomnia with anxiety and the ability to control those thoughts, we aimed to develop and evaluate the psychometric properties of the Turkish adaptation of the ICS. We also aimed to investigate the relationships between catastrophizing thoughts, general anxiety and thought control capability. The ICS has not been adapted to any Eastern or Middle Eastern society. This study was performed to analyze the factor structure of the ICS in Turkish society. The present study evaluates whether the factor structure of the ICS remains the same in Turkish society, which is culturally different from the society in which the original ICS was developed. Moreover, this study will provide Turkish mental health professionals with a cognitive theory-based instrument for the assessment of insomnia catastrophizing.

\section{Materials and methods}

\section{Pilot study}

\section{Participants}

The study group consisted of 57 Turkish young adults. There were $50(87.7 \%)$ females and $7(12.3 \%)$ males. The mean age of the participants was 23.16 years $(\mathrm{sd}=3.50)$. The participants consisted of 35 (61.4\%) university students and 22 $(38.6 \%)$ graduates.

\section{Main study}

\section{Participants}

The study group consisted of 330 Turkish young adults. There were 236 (71.5\%) females and 94 (28.5\%) males. The mean age of the participants was 21.35 years $(\mathrm{sd}=3.02$, range $=17-34$ ). The highest number of participants 194 (58.8\%) were people between the ages of 18 and 21 . In addition, $4(1.2 \%)$ of the participants are the age of 17,92 (27.9\%) between the ages of 22 and 24 , and $40(12.1 \%)$ of them between the ages of 25 and 34. The participants consisted of $278(84.2 \%)$ university students and $52(15.8 \%)$ 
graduates. In the participant group, there are mostly students from Recep Tayyip Erdoğan University $(N=80,92.7 \%)$, followed by students from Eskişehir Osmangazi University $(N=94,41.7 \%)$. Also, participants were surveyed from seven different geographical regions of the Republic of Turkey. They were primarily from Central Anatolia $(N=152$, $46.1 \%)$, followed by the Black Sea region $(N=117,35.5 \%)$. Participants answered to sleep quality in the last month on a scale of $1-10$. The mean sleep quality was $4.88(\mathrm{sd}=2.18)$. This rate is close to the midpoint. However, this rate is remarkable for the coronavirus disease 2019 (COVID-19) pandemic. Moreover, we asked the participants to indicate their daily sleep time in the last month. The mean sleep time was $7.47(\mathrm{sd}=1.50)$. Studies are stating that the average sleep time for young adults should be between 7 and $9 \mathrm{~h}$ $[28,29,30]$.

\section{Instruments}

\section{The insomnia catastrophizing scale (ICS)}

The original version of the ICS was developed in 2012 [17]. The scale consists of two subscales. The Insomnia Catastrophizing Scale-Nighttime (ICS-N) is a self-reported scale that detects catastrophizing thoughts about nighttime symptoms of insomnia. The ICS-N consists of eleven items and one dimension. Item examples include "My poor sleep will always continue" and "My poor sleep will result in a nervous breakdown". Responses to the items on the ICS-N are given based on a 6-point Likert-type scale ( $0=$ never, $5=$ always). Cronbach's alpha $(\alpha)$ was 0.92 . Item factor loadings for the ICS-N were a minimum of 0.67 for the first study and 0.65 for the second study. The variance explanation power of the ICS-N was 59.1\% [12]. The Insomnia Catastrophizing Scale-Daytime (ICS-D) is a self-reported scale that detects catastrophizing thoughts about daytime symptoms of insomnia. The ICS-D consists of six items and one dimension. Item examples include "I will feel awful all day" and "I will lose control over my ability to function". The responses to the items on the ICS-D are also given based on a 6-point Likert-type scale $(0=$ never, $5=$ always $)$. Cronbach's alpha $(\alpha)$ was 0.91 . Item factor loadings for the ICS-N were a minimum of 0.74 for both studies. The variance explanation power of the ICS-N was 70.1\% [12].

\section{Thought control capability questionnaire (TCCQ)}

The original version of the Thought Control Capability Questionnaire (TCCQ) was developed in 2005 [31]. There is a short version of the TCCQ, which has 8 items, and a long version, which has 25 items. The Turkish version of the TCCQ was adapted for the youth and adult populations in
2020. The TCCQ measures the individual's ability to control their thoughts. The responses to the items on the TCCQ are given on a 5-point Likert-type scale $(1=$ strongly disagree, $5=$ strongly agree). Cronbach's alpha $(\alpha)$ was 0.93 [32].

\section{Generalized anxiety disorder-7 scale (GADS)}

The original version of the Generalized Anxiety Disorder-7 Scale (GADS) was developed in 2006 [33]. The Turkish version of the GADS was adapted for the adult and clinical populations in 2013. The GADS is a self-reported scale that detects generalized anxiety disorder. The GADS has 7 items. The responses to the items on the GADS are given on a 4-point Likert-type scale ( $0=$ never, $3=$ nearly every day). Cronbach's alpha $(\alpha)$ was 0.85 [34].

\section{Procedure}

We communicated with the author of the ICS via e-mail and obtained permission to translate the ICS into Turkish. Later, we applied for approval from the ethics committee. We followed the steps for translating the ICS into Turkish that were suggested by the International Test Commission [35]. The instrument was designed online using Google Forms. The pilot study and Study 1 were completed between December 2020 and January 2021. All participants read and signed the informed consent form and voluntarily participated in the study.

\section{Data analysis}

The data were analyzed using SPSS [36], JASP [37], and LISREL [38]. Cronbach's alpha and McDonald's omega were calculated to assess reliability. Pearson's correlation was used to detect the relationships between variables. The factor structure was confirmed by confirmatory factor analysis (CFA). Reliability values above 0.70 , Pearson's correlation coefficients greater than 0.30 , fit values $\chi^{2 / d f}$ (chi-square/ degree of freedom) less than 5.0 [39], RMSEA values less than 0.10 [40], CFI values greater than 0.90 [41], TLI values greater than 0.95 [42], GFI values greater than 0.90 [43] and SRMR values less than 0.05 [42] were the considered criteria.

\section{Results}

\section{Translation process}

During the adaptation process, we consulted 7 experts (2 English teachers and 5 academics) when translating the original ICS into Turkish. Five experts evaluated the translations of the Turkish items and decided on the most appropriate 
forms of expression. The Turkish form was translated back into English by two linguists, and the researchers compared the items between the original English form. After the final evaluation, the ICS was administered to a small group.

\section{Pilot study}

In the pilot study, we checked the item-total correlations, reliability, and correlation between the ICS-N and ICS-D. The results showed that item-total correlations were between 0.46 and 0.89 for the ICS-N and between 0.85 and 0.93 for the ICS-D. Cronbach's alpha $(\alpha)$ was 0.90 for the ICS-N and 0.95 for the ICS-D. The ICS-N score was found to be positively correlated with the ICS-D score $(r=0.71$, $p<0.01)$. After examining the findings in the pilot study and consulting the opinions of the participants about the items, the final versions of the ICS forms were developed.

\section{Main study}

\section{Reliability}

We calculated the Cronbach's alpha $(\alpha)$ and McDonald's omega $(\omega)$ values using JASP. Cronbach's alpha $(\alpha)$ was 0.91 for the ICS-N and 0.95 for the ICS-D. McDonald's omega $(\omega)$ was 0.92 for the ICS-N and 0.95 for the ICS-D (see Table 1).

\section{Confirmatory factor analysis}

CFA was performed with LISREL version 8.80 to evaluate the fit indices using maximum likelihood estimation. The ICS-N were tested, and the results were as follows: $\chi^{2}=192.03, d f=44, \mathrm{GFI}=0.90, \mathrm{CFI}=0.97, \mathrm{SRMR}=0.05$, $\mathrm{TLI}=0.96$, and RMSEA $=0.10$. The model suggested significant modifications between item-3 and item-5. The error terms of item-3 (My physical health will be negatively affected) and item-5 (My poor sleep will have serious consequences) in the ICS-N were associated. Therefore, we modified item-3 and item-5. After the modifications, the results were as follows: $\chi^{2}=155.76, d f=43, \mathrm{GFI}=0.92, \mathrm{CFI}=0.98$, $\mathrm{SRMR}=0.04, \mathrm{TLI}=0.97$, and RMSEA $=0.09$. Additionally, ICS-D were tested, and the results were as follows: $\chi^{2}=87.85, d f=9, \mathrm{GFI}=0.92, \mathrm{CFI}=0.98, \mathrm{SRMR}=0.03$, $\mathrm{TLI}=0.96$, IFI $=0.98$, and RMSEA $=0.16$. The model suggested significant modifications between item-2 and item-3. The error terms of item-2 (My poor sleep will have serious consequences today) and item-3 (I will feel worse and worse) in the ICS-D were associated. Thus, we modified item-2 and item-3. After the modifications, the results were as follows: $\chi^{2}=36.94, d f=8$, GFI $=0.96, \mathrm{CFI}=0.99$, SRMR $=0.02, \mathrm{TLI}=0.98$, and RMSEA $=0.10$. In this study, the factors loadings for the ICS-N and ICS-D were high, which was also observed in previous studies. Item factor loads were found to be at least 0.51 for the ICS-N and at least 0.85 for the ICS-D (see Table 2).
Table 1 Item analysis and reliability

\begin{tabular}{lrrllll}
\hline & Mean & $S D$ & $\begin{array}{l}\text { Item-total cor- } \\
\text { relations }\end{array}$ & $\begin{array}{l}\text { Item-rest cor- } \\
\text { relations }\end{array}$ & If item dropped & \\
\cline { 6 - 7 } & & & & & Cronbach's $\alpha$ & McDonald's $\omega$ \\
\hline ICS_N1 & 2.16 & 1.44 & $0.65^{*}$ & $0.58^{*}$ & 0.91 & 0.91 \\
ICS_N2 & 1.96 & 1.54 & $0.64^{*}$ & $0.57^{*}$ & 0.91 & 0.91 \\
ICS_N3 & 2.92 & 1.66 & $0.77^{*}$ & $0.71^{*}$ & 0.90 & 0.91 \\
ICS_N4 & 2.50 & 1.55 & $0.58^{*}$ & $0.50^{*}$ & 0.91 & 0.92 \\
ICS_N5 & 2.42 & 1.67 & $0.79^{*}$ & $0.73^{*}$ & 0.90 & 0.91 \\
ICS_N6 & 2.79 & 1.76 & $0.75^{*}$ & $0.69^{*}$ & 0.91 & 0.91 \\
ICS_N7 & 2.04 & 1.65 & $0.86^{*}$ & $0.82^{*}$ & 0.90 & 0.90 \\
ICS_N8 & 2.54 & 1.68 & $0.76^{*}$ & $0.69^{*}$ & 0.91 & 0.91 \\
ICS_N9 & 2.10 & 1.69 & $0.83^{*}$ & $0.78^{*}$ & 0.90 & 0.90 \\
ICS_N10 & 1.76 & 1.68 & $0.69^{*}$ & $0.62^{*}$ & 0.91 & 0.91 \\
ICS_N11 & 2.92 & 1.64 & $0.72^{*}$ & $0.65^{*}$ & 0.91 & 0.91 \\
ICS-Nighttime & 26.11 & 13.18 & - & - & 0.92 & 0.91 \\
ICS_D1 & 2.59 & 1.62 & $0.89^{*}$ & $0.84^{*}$ & 0.95 & 0.95 \\
ICS_D2 & 2.24 & 1.54 & $0.90^{*}$ & $0.85^{*}$ & 0.94 & 0.94 \\
ICS_D3 & 2.16 & 1.61 & $0.91^{*}$ & $0.86^{*}$ & 0.94 & 0.94 \\
ICS_D4 & 2.64 & 1.55 & $0.92^{*}$ & $0.88^{*}$ & 0.94 & 0.94 \\
ICS_D5 & 2.67 & 1.60 & $0.90^{*}$ & $0.85^{*}$ & 0.94 & 0.95 \\
ICS_D6 & 2.50 & 1.59 & $0.89^{*}$ & $0.84^{*}$ & 0.95 & 0.95 \\
ICS-Daytime & 14.80 & 8.56 & - & - & 0.95 & 0.95 \\
\hline
\end{tabular}

${ }^{*} p<0.01$ 
Table 2 Factor loadings

\begin{tabular}{clllll}
\hline & Original study & Italian study & $\begin{array}{l}\text { Portuguese } \\
\text { study }\end{array}$ & Turkish study & \\
\cline { 5 - 6 } & & & & & No modification \\
& & & & A-modification \\
\hline ICS-Nighttime & & 0.78 & 0.72 & 0.60 & 0.60 \\
ICS_N1 & 0.72 & 0.70 & 0.73 & 0.59 & 0.59 \\
ICS_N2 & 0.67 & 0.80 & 0.76 & 0.74 & 0.71 \\
ICS_N3 & 0.82 & 0.65 & 0.61 & 0.51 & 0.51 \\
ICS_N4 & 0.70 & 0.84 & 0.80 & 0.77 & 0.75 \\
ICS_N5 & 0.84 & 0.66 & 0.73 & 0.72 & 0.72 \\
ICS_N6 & 0.76 & 0.84 & 0.81 & 0.87 & 0.88 \\
ICS_N7 & 0.77 & 0.72 & 0.63 & 0.74 & 0.73 \\
ICS_N8 & 0.74 & 0.78 & 0.81 & 0.83 & 0.84 \\
ICS_N9 & 0.77 & 0.78 & 0.74 & 0.65 & 0.66 \\
ICS_N10 & 0.65 & 0.75 & 0.75 & 0.68 & 0.67 \\
ICS_N11 & 0.71 & & & & 0.86 \\
ICS-Daytime & & 0.76 & 0.85 & 0.86 & 0.85 \\
ICS_D1 & 0.80 & 0.87 & 0.90 & 0.87 & 0.86 \\
ICS_D2 & 0.84 & 0.82 & 0.81 & 0.88 & 0.92 \\
ICS_D3 & 0.74 & 0.89 & 0.89 & 0.91 & 0.89 \\
ICS_D4 & 0.83 & 0.91 & 0.91 & 0.88 & \\
ICS_D5 & 0.84 & 0.85 & 0.84 & 0.86 & \\
ICS_D6 & 0.76 & & & & \\
\hline
\end{tabular}

\section{Convergent validity}

The correlations among the ICS-N, ICS-D, TCCQ and GADS were analyzed using Pearson's correlation analysis. The ICS-N score was found to be positively correlated with the ICS-D score $(r=0.74, p<0.01)$ and the GADS score $(r=0.51, p<0.01)$. The ICS-N score was found to be negatively correlated with the TCCQ score $(r=-0.58, p<0.01)$. The ICS-D was found to be positively correlated with the GADS score $(r=0.46, p<0.01)$ and negatively correlated with the TCCQ score $(r=-0.50, p<0.01)$.

\section{Sex differences in the ICS-D and ICS-N}

In the present study, we compared the ICS-N and ICS-D scores between females and males using SPSS v.18 software. Independent sample $t$-tests showed that both the ICS-N and ICS-D scores were significantly higher for females than for males (see Table 3).

\section{Discussion}

In the present study, we tested the Turkish adaptation of the ICS-D and ICS-N in a young adult population. First, we translated the instrument. Then, we performed a pilot study and a main study. In the pilot study, we tested the itemtotal correlations for both the ICS-D and ICS-N, reliability scores, and correlations between the ICS-D and ICS-N. The findings of the pilot study showed good item-total correlations, high reliability, and strong correlations between the ICS-D and ICS-N. The findings of the main study showed similar reliability scores for the ICS-N and ICS-D as those in the pilot study. In the main study, the ICS-N scores were $26.11(\mathrm{sd}=13.18)$, and the ICS-D scores were 14.80 $(\mathrm{sd}=8.56)$. The factor structures of the ICS-D and ICS-N were confirmed in a Turkish population. The item factor loads were also satisfactory. Pearson's correlations among the ICS-N, ICS-D, TCCQ, and GADS were at least moderate. Moreover, females had higher scores than males on both the ICS-N and ICS-D.

Table 3 Sex differences of ICS

\begin{tabular}{|c|c|c|c|c|c|c|}
\hline \multirow[t]{2}{*}{ Parameter } & \multicolumn{2}{|l|}{ Male } & \multicolumn{2}{|c|}{ Female } & \multirow[t]{2}{*}{$t(328)$} & \multirow[t]{2}{*}{$p$} \\
\hline & M & $S D$ & $\mathrm{M}$ & $S D$ & & \\
\hline ICS-N & 23.46 & 12.61 & 27.17 & 13.28 & -2.321 & $0.021^{*}$ \\
\hline ICS-D & 13.22 & 8.79 & 15.43 & 8.40 & -2.123 & $0.035^{*}$ \\
\hline
\end{tabular}

$* p<0.05$, Cohen's $d$ for ICS-N $=-0.28$ and ICS $-\mathrm{D}=-0.26$ 
The ICS-D and ICS-N have high reliability scores (greater than 0.90), as in the original studies [12, 17], the Italian version [7] and the Portuguese version [19]. The ICS-D and ICS-N factor structures in the Italian [7] and Portuguese [19] adaptations were confirmed to be the same as the single-factor original structure. Similarly, in the present study, the single-factor structure was confirmed, and the fit indices showed that the data fit the model. Item factor loadings ranged from 0.51 to 0.88 for the ICS-N and between 0.85 and 0.92 for the ICS-D. Item factor loads should be above 0.30 [44]. The Turkish versions of the ICS-D and ICS-N met the minimum item factor loadings condition. In previous studies, moderate [17] and strong [12] correlations were provided between ICS-D, ICS-N, and anxiety. In the present study, the ICS-D and ICS-N had moderate correlations with generalized anxiety and thought control capability, which is evidence supporting convergent validity.

The data of the current study were provided during the COVID-19 pandemic. It is noteworthy that in the present study the ICS-D score was 14.80 and the ICS-N score was 26.11. In the original study, participants with insomnia disorder had an ICS-D score of 6.40 and an ICS-N score of 14.9 [12]. More clearly, the ICS scores in current study are higher than in the original scale study. This finding may be because the adaptation studies of the scale were carried out during the COVID-19 pandemic. Because studies have shown that the prevalence of insomnia, length of stay in bed, and total sleep time increased significantly in the general population during the COVID19 pandemic $[45,46]$ and that the population in the quarantine process had higher levels of depression, anxiety, and more than the normal population demonstrated a risk of insomnia [47]. It appears that the COVID-19 pandemic is associated with higher rates of insomnia than normaltime (before COVID-19 pandemic) insomnia and that the pandemic causes various mental problems [48]. We think that the strong relationship observed between the COVID19 outbreak and insomnia symptoms [49], which points to higher scores than those observed in the general population, explains the insomnia catastrophe scores observed in the current study, which were higher than individuals with insomnia disorders.

A remarkable finding of the present study was that females had higher ICS-D and ICS-N scores than males. Insomnia catastrophizing is a new concept; therefore, the studies examining insomnia catastrophizing by sex are limited. Although some differences have been reported in Italy [7], more research is needed on the differences in insomnia catastrophizing by sex.

\section{Limitations}

This study has some limitations. The first of these limitations is that the Turkish version of the ICS was only tested in a young adult group. Second, we did not determine the cutoff score. Increasing ICS scores indicate more insomnia catastrophizing. Third, we could not use the Insomnia Severity Scale [20] in the present study. Because although we sent e-mails to the authors of the scale many times, we could not get permission to use the scale. In future studies, researchers should evaluate the relationships between the ICS-D and ICS-N forms and insomnia severity. Fourth, test-retest validity could not be assessed. Fifth, we did not test measurement models for sex differences or investigate possible relationships.

\section{Conclusion}

The findings showed that the ICS-D and ICS-N are valid and reliable instruments in the young adult population. ICS-D and ICS-N can be used to measure insomnia catastrophizing in Turkish culture. Cognitive-behavioral therapy and psychotherapy are the most important treatment modalities in Turkey [50]. Mental health professionals (such as psychological counselors and psychologists) can use these instruments in individual counseling, therapy, and group therapy. Specifically, cognitive or cognitivebehavioral therapists can detect individuals' insomnia catastrophizing using these instruments. As a result, therapists can make significant contributions to their clients' progress in therapy by enabling them to change their cognitive structures (both daytime and nighttime) regarding the future and the present. Second, adaptations of the ICS to Italian [7] and Portuguese [19] have been reported. The present study provides evidence that the cognitive structure of insomnia catastrophizing can be confirmed in societies with culturally significant differences. This study encourages the adaptation of the ICS for use in different cultures. It is important to investigate the cause of sex differences in ICS scores in Turkey and possible differences in other cultures (such as Middle Eastern and Eastern societies). It is important to consider the reasons females experience higher levels of insomnia catastrophizing and develop interventions to reduce insomnia catastrophizing.

Supplementary Information The online version contains supplementary material available at https://doi.org/10.1007/s41105-021-00336-7.

Author contributions FK: Conceptualization, FK, OM, S Y-K, YEE and BB: Methodology, FK, OM, S Y-K, YEE and BB: Formal analysis and investigation, FK, OM, S Y-K, YEE and BB: Writing-original 
draft preparation, FK, OM, S Y-K, YEE and BB: Writing-review and editing, FK, OM, S Y-K, YEE and BB: Resources

Funding None.

\section{Declarations}

Conflict of interest Fedai Kabadayi, Oğuz Mercan, Sema YaziciKabadayi, Yaser Emir Elhatip and Baki Büyüksevindik declare that they have no conflict of interest.

Ethical approval This study was approved by the Research Ethics Committee of Recep Tayyip Erdogan University (Ethics Approval Number: 2020/157, December 8, 2020).

Informed consent Informed consent was obtained from all individual participants included in the study.

\section{References}

1. American Psychiatric Association. Diagnostic and statistical manual of mental disorders (DSM- $\left.{ }^{\circledR}\right)$. Arlington: American Psychiatric Association; 2013.

2. Walsh JK. Clinical and socioeconomic correlates of insomnia. J Clin Psychiatr. 2004;65:13-9.

3. Roth T, Coulouvrat C, Hajak G, et al. Prevalence and perceived health associated with insomnia based on DSM-IV-TR; international statistical classification of diseases and related health problems, tenth revision; and research diagnostic criteria/international classification of sleep disorders, criteria: Results from the America insomnia survey. Biol Psychiatr. 2011. https://doi. org/10.1016/j.biopsych.2010.10.023.

4. González-Sanguino C, Ausín B, Castellanos MÁ, et al. Mental health consequences during the initial stage of the 2020 Coronavirus pandemic (COVID-19) in Spain. Brain Behav Immun. 2020. https://doi.org/10.1016/j.bbi.2020.05.040.

5. Pappa S, Ntella V, Giannakas T, et al. Prevalence of depression, anxiety, and insomnia among healthcare workers during the COVID-19 pandemic: a systematic review and meta-analysis. Brain Behav Immun. 2020. https://doi.org/10.1016/j.bbi.2020. 05.026.

6. Duran S, Geyiktepe ÖE. Psychologic distress and sleep quality among adults in Turkey during the COVID-19 pandemic. Prog Neuro-Psychopharmacol Biol Psychiatr. 2021. https://doi.org/10. 1016/j.pnpbp.2021.110254.

7. Ballesio A, Mallia L, Cellini N, et al. Italian adaptation of the Insomnia Catastrophising Scale (ICS): a tool to evaluate insomnia-specific catastrophic thinking. Sleep Biol Rhythm. 2018. https://doi.org/10.1007/s41105-018-0177-3.

8. Quartana PJ, Campbell CM, Edwards RR. Pain catastrophizing: a critical review. Exp Rev Neurotherap. 2009. https://doi.org/10. 1586/ern.09.34.

9. DiGiuseppe RA, Doyle KA, Dryden W, et al. A practitioner's guide to rational emotive behavior therapy. 3rd ed. New York: Oxford University Press; 2013.

10. Vasey MW, Borkovec TD. A catastrophizing assessment of worrisome thoughts. Cogn Therapy Res. 1992. https://doi.org/10.1007/ BF01175138.

11. Tan LO, Hadjistavropoulos T, MacNab YC. The Catastrophic Thoughts about Insomnia Scale (CTIS): development and validation. Cogn Therapy Res. 2017. https://doi.org/10.1007/ s10608-016-9804-y.
12. Jansson-Fröjmark M, Harvey AG, Flink IK. Psychometric properties of the Insomnia Catastrophizing Scale (ICS) in a large community sample. Cogn Behav Therapy. 2020. https://doi.org/ 10.1080/16506073.2019.1588362.

13. Ali RM, Zolezzi M, Awaisu A. A systematic review of instruments for the assessment of insomnia in adults. Nat Sci Sleep. 2020. https://doi.org/10.2147/NSS.S250918.

14. Pallesen S, Bjorvatn B, Nordhus IH, et al. A new scale for measuring insomnia: the Bergen Insomnia Scale. Percep Mot Ski. 2008. https://doi.org/10.2466/pms.107.3.691-706.

15. Bastien $\mathrm{CH}$, Vallières $\mathrm{A}$, Morin $\mathrm{CM}$. Validation of the Insomnia Severity Index as an outcome measure for insomnia research. Sleep Medic. 2001. https://doi.org/10.1016/S1389-9457(00) 00065-4.

16. Soldatos CR, Dikeos DG, Paparrigopoulos TJ. Athens Insomnia Scale: validation of an instrument based on ICD-10 criteria. J Psychosom Res. 2000. https://doi.org/10.1016/S0022-3999(00) 00095-7.

17. Jansson-Fröjmark M, Harvey AG, Flink IK. Psychometric properties of the Insomnia Catastrophising Scale. J Sleep Res. 2012;21:168-168.

18. Harvey AG. A cognitive model of insomnia. Behav Res Therapy. 2002. https://doi.org/10.1016/s0005-7967(01)00061-4.

19. Marques-Correia AL. Adaptação Portuguesa da ICS (Insomnia Catastrophizing Scale) (In Portuguese). Unpublished master's thesis. 2019; Portugal: Universidade de Aveiro.

20. Boysan M, Güleç M, Besiroglu L, Kalafat T. Psychometric properties of The Insomnia Severity Index in Turkish sample. Anatolia J Psychiatr. 2010;11:248-52.

21. Bay T, Ergun A. Validity and reliability of Bergen Insomnia Scale (BIS) among adolescents. Clin Exp Health Sci. 2018;8:268-75.

22. Ağargün MY, Kara H, Anlar O. Pittsburgh Uyku Kalitesi İndeksinin geçerliği ve güvenirliği (In Turkish). Turkish J Psychiatr. 1996;7:107-15.

23. Boysan M, Merey Z, Kalafat T, Kağan M. Validation of a brief version of the Dysfunctional Beliefs and Attitudes About Sleep Scale in Turkish sample. Proc-Soc Behav Sci. 2010. https://doi. org/10.1016/j.sbspro.2010.07.095.

24. Gellis LA, Park A. Nighttime thought control strategies and insomnia severity. Cogn Therapy Res. 2013. https://doi.org/10. 1007/s10608-012-9479-y.

25. Wei $Y$, Leerssen J, Wassing R, et al. Reduced dynamic functional connectivity between salience and executive brain networks in insomnia disorder. J Sleep Res. 2020. https://doi.org/10.1111/jsr. 12953.

26. Jansson-Fröjmark M, Jacobson K. Cognitive behavioural therapy for insomnia for patients with co-morbid generalized anxiety disorder: an open trial on clinical outcomes and putative mechanisms. Behav Cogn Psychother. 2021. https://doi.org/10.1017/ S1352465821000023.

27. Sun Q, Tan L. Comparing primary insomnia to the insomnia occurring in major depression and general anxiety disorder. Psychiatr Res. 2019. https://doi.org/10.1016/j.psychres.2019.112514.

28. Hirshkowitz M, Whiton K, Albert SM, et al. National Sleep Foundation's sleep time duration recommendations: methodology and results summary. Sleep Health. 2015. https://doi.org/10.1016/j. sleh.2014.12.010.

29. Klerman EB, Dijk DJ. Interindividual variation in sleep duration and its association with sleep debt in young adults. Sleep. 2005. https://doi.org/10.1093/sleep/28.10.1253.

30. Léger D, du Roscoat E, Bayon V, et al. Short sleep in young adults: insomnia or sleep debt? Prevalence and clinical description of short sleep in a representative sample of 1004 young adults from France. Sleep Med. 2011. https://doi.org/10.1016/j.sleep. 2010.12.012. 
31. Luciano JV, Algarabel S, Tomás JM, Martínez JL. Development and validation of the Thought Control Ability Questionnaire. Personal Individ Differ. 2005. https://doi.org/10.1016/j.paid.2004.06. 020.

32. Sarıçam H, Mert A. Turkish version of the Thought Control Capability Questionnaire: a scale adaptation study. Mersin Uni J Fac Edu. 2020. https://doi.org/10.17860/mersinefd.619831.

33. Spitzer RL, Kroenke K, Williams JB, Löwe B. A brief measure for assessing generalized anxiety disorder: the GAD-7. Arch Inter Medic. 2006. https://doi.org/10.1001/archinte.166.10.1092.

34. Konkan R, Şenormanci Ö, Güçlü O, et al. Validity and reliability study for the Turkish adaptation of the Generalized Anxiety Disorder-7 (GAD-7) Scale. Arch Neuropsychol. 2013. https://doi.org/ 10.4274/npa.y6308.

35 International Test Commission. ITC guidelines for translating and adapting (second edition). Int J Test. 2017. https://doi.org/ 10.1080/15305058.2017.1398166.

36. Pasw SPSS. Statistics 18 core system user's guide. Chicago: SPSS Inc; 2019.

37. Love J, Selker R, Marsman M, et al. JASP: graphical statistical software for common statistical designs. J Stat Softw. 2019. https://doi.org/10.18637/jss.v088.i02.

38. Jöreskog KG, Sörbom D. LISREL 8.80 for windows. Lincolnwood: Scientific Software International Inc.; 2006.

39. Marsh HW, Hocevar D. Application of confirmatory factor analysis to the study of self-concept: first-and higher order factor models and their invariance across groups. Psych Bull. 1985. https:// doi.org/10.1037/0033-2909.97.3.562.

40. Kline RB. Principles and practice of structural equation modeling. London: Guilford publications; 2016.

41. Bentler PM. Comparative fit indexes in structural models. Psych Bull. 1990. https://doi.org/10.1037/0033-2909.107.2.238.

42. Hu LT, Bentler PM. Cutoff criteria for fit indexes in covariance structure analysis: conventional criteria versus new alternatives. Struct Equal Model. 1999. https://doi.org/10.1080/1070551990 9540118.
43. Jöreskog KG, Sörbom D. LISREL 8: structural equation modeling with the SIMPLIS command language. London: Scientific Software International; 1993.

44. Seçer İ. Psychological test development and adaptation process: SPSS and LISREL practices (In Turkish). Ankara: An1 Publishing; 2015.

45. Li Y, Qin Q, Sun Q, et al. Insomnia and psychological reactions during the COVID-19 outbreak in China. J of Clin Sleep Med. 2020. https://doi.org/10.5664/jcsm.8524.

46. Huang Y, Wang Y, Zeng L, et al. Prevalence and correlation of anxiety, insomnia and somatic symptoms in a Chinese population during the COVID-19 epidemic. Front Psychiatry. 2020. https:// doi.org/10.3389/fpsyt.2020.568329.

47. Wang C, Song W, Hu X, et al. Depressive, anxiety, and insomnia symptoms between population in quarantine and general population during the COVID-19 pandemic: a case-controlled study. BMC Psychiatry. 2021. https://doi.org/10.1186/ s12888-021-03108-2.

48. Killgore WD, Cloonan SA, Taylor EC, et al. Suicidal ideation during the COVID-19 pandemic: the role of insomnia. Psychiatry Res. 2020. https://doi.org/10.1016/j.psychres.2020.113134.

49. Cheng P, Casement MD, Kalmbach DA, et al. Digital cognitive behavioral therapy for insomnia promotes later health resilience during the coronavirus disease 19 (COVID-19) pandemic. Sleep. 2021. https://doi.org/10.1093/sleep/zsaa258.

50. Yorulmaz O, Sapmaz-Yurtsever S, Gökdag C, et al. The future of psychotherapy in Turkey: predictions for the next 10 years. $\mathrm{J}$ Nerv Ment Dis. 2018. https://doi.org/10.1097/NMD.0000000000 000797.

Publisher's Note Springer Nature remains neutral with regard to jurisdictional claims in published maps and institutional affiliations. 